\title{
Saliva as a biomarker for head and neck squamous cell carcinoma: In vitro detection of cytotoxic effects by using the plating efficiency index
}

\author{
MARC B. BLOCHING ${ }^{1}$, JAMES BARNES ${ }^{2}$, WOLFRAM AUST ${ }^{1}$, STEPHAN KNIPPING $^{3}$, \\ KATRIN NEUMANN ${ }^{3}$, TAMARA GRUMMT ${ }^{4}$ and RAMIN NAIM ${ }^{1}$
}

\begin{abstract}
${ }^{1}$ Department of Otolaryngology, Head and Neck Surgery, University of Homburg/Saar, Germany; ${ }^{2}$ Warwick Medical School, University of Warwick, Coventry, CV4 7AL, UK; ${ }^{3}$ Department of Otolaryngology, Head and Neck Surgery, Martin Luther University Halle-Wittenberg; ${ }^{4}$ The Federal Environmental Agency of Germany, Berlin, Germany
\end{abstract}

Received April 27, 2007; Accepted July 2, 2007

\begin{abstract}
Worldwide, the incidence of head and neck squamous cell carcinoma (HNSCC) is increasing. The development of an effective prevention program would provide a promising opportunity to control this disease. We applied the 'plating efficiency index' of Chinese hamster lung fibroblasts to demonstrate the presence of cytotoxic effects in saliva samples from cancer patients as well as from healthy probands. Correlations between individual risk factors and the cytotoxic effects of saliva specimens were analysed and evaluated. Saliva samples were obtained from male patients $(n=43)$ with carcinomas of the upper aerodigestive tract, and from a healthy age-matched male control group $(n=131)$ with different tobacco and alcohol consumption habits. The extraction of non-stimulated sober saliva was chosen for this investigation. In vitro cytotoxicity of the saliva was detected using the permanent mammalian cell line V79 (lung fibroblasts) of Chinese hamsters. The determination of the plating efficiency index was made in the logarithmic growth phase of the initial cell culture. A reduction in cell colonies (plating efficiency) of at least 50\% was defined as strongly cytotoxic. A significant direct correlation was found between daily tobacco consumption and the reduction of plating efficiency $(\mathrm{p}<0.0001)$. We found only a moderate increase in the cytotoxicity of tested saliva samples in correlation with daily alcohol uptake. The difference between tumor patients and healthy probands was highly significant ( $\chi^{2}$ test; $\left.p=0.001\right)$. Using the method of logistic regression analysis, we found a 3.6-fold increased
\end{abstract}

Correspondence to: Dr Marc B. Bloching, Department of Otolaryngology, Head and Neck Surgery, University of Homburg/ Saar, Kirrberger Strasse, 66421 Homburg/Saar, Germany

E-mail: hnomblo@uks.eu

Key words: plating efficiency index, biomarker, head and neck squamous cell carcinoma, saliva, cytotoxicity, upper aerodigestive tract cancer risk in probands with cytotoxic saliva $(\mathrm{p}<0.001)$. According to our results, the plating efficiency index seems to be a suitable method for the detection of increased cancer risk. In combination with several effective biomarkers on cytogenetic end-points, it may help to establish biomonitoring programs for secondary cancer prevention.

\section{Introduction}

Primary cancers of the head and neck region have varying types of histology. Squamous cell carcinomas (SCCs) are among the most common malignancies of the upper aerodigestive tract (1). The incidence of these tumors varies worldwide on the basis of changing 'lifestyle' factors $(2,3)$ such as chronic tobacco and alcohol consumption or changing exposure to environmental carcinogens. Despite multimodal therapies with often mutilating surgical procedures (especially in extended tumor stages), the prognosis for these tumors remains poor. Hence, the development of effective prevention programs would provide a promising opportunity to control this increasingly common disease.

In general, there are two different methods of disease prevention. First, and most effective, is the reduction of avoidable exogenous risk factors. The second involves the identification of people bearing individual increased cancer risk, which may help in the detection of early-stage carcinomas (4-7). Hopefully, this will be achieved in the future through the use of biomarkers showing biological effects, like permanent changes at the genetic end-point or different individual susceptibilities. With this in mind, we examined whether saliva may be used as a predictive indicator to measure the carcinogenic milieu of the upper aerodigestive tract. We applied the 'plating efficiency index' of Chinese hamster lung fibroblasts to assess the cytotoxic effects of saliva samples from healthy probands and cancer patients.

The cytotoxic effects of saliva may be affected by genetic factors (e.g. polymorphisms of enzymes) which are involved in the toxification and detoxification of different agents or metabolites. In particular, polymorphisms of the p450-system, glutathione-S-transferases and the alcohol-dehydrogenasesystem have been shown to influence carcinogenesis in the 
upper aerodigestive tract (8-11). Therefore, in this study the correlations between individual risk factors and the cytotoxic effects of saliva specimens were analysed in order to evaluate a potential new method in predictive oncology.

\section{Materials and methods}

Donors. Saliva samples were obtained from male patients $(n=43)$ with carcinomas of the upper aerodigestive tract, and from a healthy age-matched male control group $(n=131)$ with different tobacco and alcohol consumption habits. For population monitoring, each patient and each control group member completed a questionnaire detailing age, sex, medical history, smoking and drinking habits and other carcinogenic/mutagenic agents which could influence cancer progression (organic solvents, asbestos, petrol, heavy metals, dusts in general, radiation) (12).

The study design was approved by the Ethics Commission of the medical department of the Martin Luther University Halle-Wittenberg. The ethical standards of the Declaration of Helsinki were observed.

Extraction of saliva specimens. The extraction of nonstimulated sober saliva was chosen for this investigation. In order to exclude local toxic effects, the study participants were instructed to not eat, smoke or drink for $12 \mathrm{~h}$ before the sampling of the specimen (normal dental care was, however, maintained). Due to strong biological contamination, the specimens were filtered through a $0.2 \mu \mathrm{m}$ polycarbonate filter and stored at $-20^{\circ} \mathrm{C}$.

Assessment of cytotoxicity. The in vitro cytotoxicity of the saliva was detected using the permanent mammalian cell line V79 (lung fibroblasts) of Chinese hamsters (Cricetulus griseus). This cell line is characterised by fast growth and stable karyotype (13). The cells were incubated with Dulbecco's modified Eagle's medium in $25 \mathrm{ml}$ cell culture bottles at a temperature of $37^{\circ} \mathrm{C}$ and in an atmosphere of $5 \%$ $\mathrm{CO}_{2}$ with $95 \%$ relative humidity. Directly before cultivation, $0.584 \mathrm{~g}$ L-glutamine, 10 units penicillin, $10 \mu \mathrm{g}$ streptomycin, $25 \mathrm{pg}$ amphotericin B and fetal calf serum in an end concentration of $10 \%$ to the culturing medium (Sigma, Deisenhofen, Germany) were added. Determination of the plating efficiency index was made in the logarithmic growth phase of the initial cell culture. After trypsinating and washing, the viability of the cells was determined using trypan blue staining (not $<80 \%$ ). At the next step, the cells were seeded at a low density of $\sim 100$ cells per culture bottle to prevent cell-to-cell contacts. After cell adhesion ( 2-3 h), $1 \mathrm{ml}$ of saliva specimen was added to the solution. In a parallel test, distilled water served as a negative control. Cultures with both the saliva and the negative controls were incubated for 7-10 days. Due to the size and morphology of the cell colonies, the culture medium was decanted and the cells fixed with methano-acetic acid solution (3:1). Before being manually counted, the cells were stained with Giemsa (2\%) for $30 \mathrm{~min}$. A reduction in cell colonies (plating efficiency) of at least $50 \%$ in the saliva testing compared to the concurrent negative control was defined as strong cytotoxicity. A slight reduction of $\sim 20-50 \%$ in the growing colonies was determined
Table I. Characterisation of study participants concerning age, gender, tumor localisation and smoking habits.

\begin{tabular}{lcc}
\hline$(\mathrm{n}=174)$ & Patients & Healthy probands \\
\hline Number (n) & 43 & 131 \\
Mean age (years) & 52.6 & 44.3 \\
Gender & & \\
$\quad$ Male & 40 & 131 \\
Female & 3 & - \\
Smokers & 31 & 36 \\
Former smokers & 11 & 42 \\
Non-smokers & 1 & 53 \\
Laryngeal SCC & 17 & - \\
Hypopharyngeal SCC & 7 & - \\
Oropharyngeal SCC & 19 & \\
\hline
\end{tabular}

Table II. Characterisation of study participants concerning individual tobacco and alcohol consumption.

\begin{tabular}{lccc}
\hline$(\mathrm{n}=174)$ & $\begin{array}{c}\text { Abstinent } \\
(\%)\end{array}$ & $\begin{array}{c}\leq 25 \mathrm{~g} \text { alcohol/ } \\
\text { day }(\%)\end{array}$ & $\begin{array}{c}>25 \mathrm{~g} \text { alcohol/ } \\
\text { day }(\%)\end{array}$ \\
\hline Non-smokers & $13(7.5)$ & $21(12.1)$ & $20(11.5)$ \\
$\leq 10 \mathrm{cig} /$ day & $1(0.6)$ & $1(0.6)$ & $12(6.9)$ \\
$10-20 \mathrm{cig} /$ day & $4(2.3)$ & $4(2.3)$ & $30(17.2)$ \\
$>20 \mathrm{cig} /$ day & $1(0.6)$ & $3(1.7)$ & $12(6.9)$ \\
Former smokers & $11(6.3)$ & $10(5.7)$ & $31(17.8)$ \\
\hline
\end{tabular}

to be only moderate cytotoxicity. The test battery was repeated three times to get reproducible results.

Statistical analysis. The SPSS software pack was used for statistical analysis. First, we compared the plating efficiency index of patients and healthy probands using descriptive methods. Correlations between the plating efficiency index and smoking and drinking habits were analysed using the Mann-Whitney U test. The Bonferroni method was performed to correct for multiple testing. Differences between the biological effects of the saliva specimens from the patients and the healthy probands were tested with the $\chi^{2}$ test. Individual cancer risk (odds ratio) was predicted in relation to the plating efficiency index and the smoking and drinking habits of the study participants using multiple logistic regression analysis. The overall level of statistical relevance used was 0.05 . 


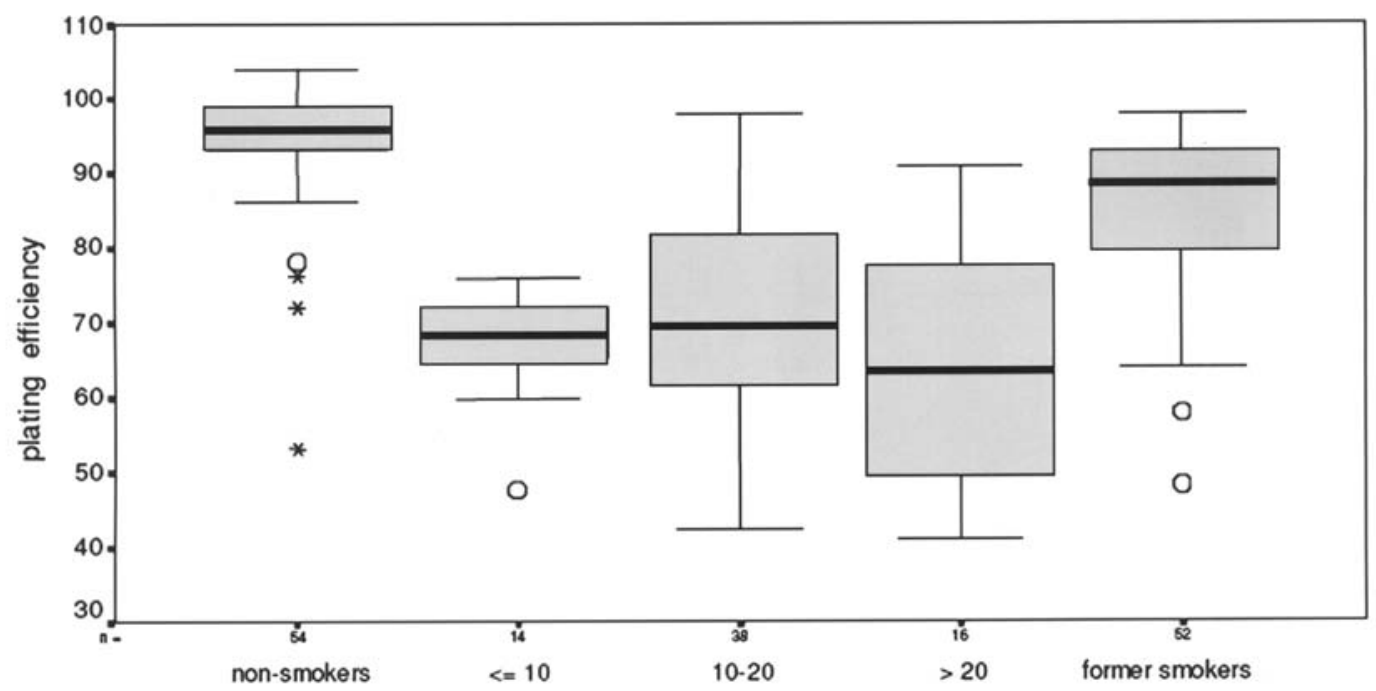

Figure 1. Boxplot diagram to visualise the reduction of plating efficiency in correlation to daily tobacco consumption; detailed results (p-values) are listed in Table III.

Table III. P-values to prove the different biological effects (i.e. cytotoxicity) as measured by the plating efficiency of tested saliva samples in correlation to individual tobacco consumption.

\begin{tabular}{lcccc}
\hline & Non-smokers & Former smokers & $\leq 10 \mathrm{cig} / \mathrm{day}$ & $10-20 \mathrm{cig} / \mathrm{day}$ \\
\hline Non-smokers & - & & \\
Former smokers & $\mathrm{p}<0.0001^{\mathrm{a}}$ & - & - & \\
$\leq 10$ cig/day & $\mathrm{p}<0.0001^{\mathrm{a}}$ & $\mathrm{p}<0.0001^{\mathrm{a}}$ & $\mathrm{p}=0.556$ & - \\
$10-20$ cig/day & $\mathrm{p}<0.0001^{\mathrm{a}}$ & $\mathrm{p}<0.0001^{\mathrm{a}}$ & $\mathrm{p}=0.697$ & $\mathrm{p}=0.251$ \\
$>20$ cig/day & $\mathrm{p}<0.0001^{\mathrm{a}}$ & $\mathrm{p}<0.0001^{\mathrm{a}}$ & & \\
\hline
\end{tabular}

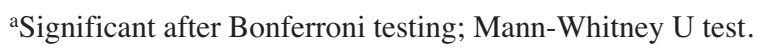

\section{Results}

The characteristics of the study participants with regard to age, gender, smoking habits and tumor localisation are listed in Table I. In addition, the individual daily tobacco and alcohol consumption of all donors is summarised in Table II.

The direct comparison of daily tobacco consumption and the reduction of plating efficiency significantly demonstrates the hazardous biological effects of chronic tobacco abuse. The effects are shown in Fig. 1. Table III shows the detailed results of the statistical analysis of differences between study participants with different smoking habits.

In contrast to the influence of smoking habits on the biological effects of saliva, we found only a moderate increase in the cytotoxicity of the tested saliva samples in correlation to the daily alcohol uptake. This slight tendency is shown in Fig. 2 (Mann-Whitney U test; Bonferroni testing; $\mathrm{p}>0.05)$.

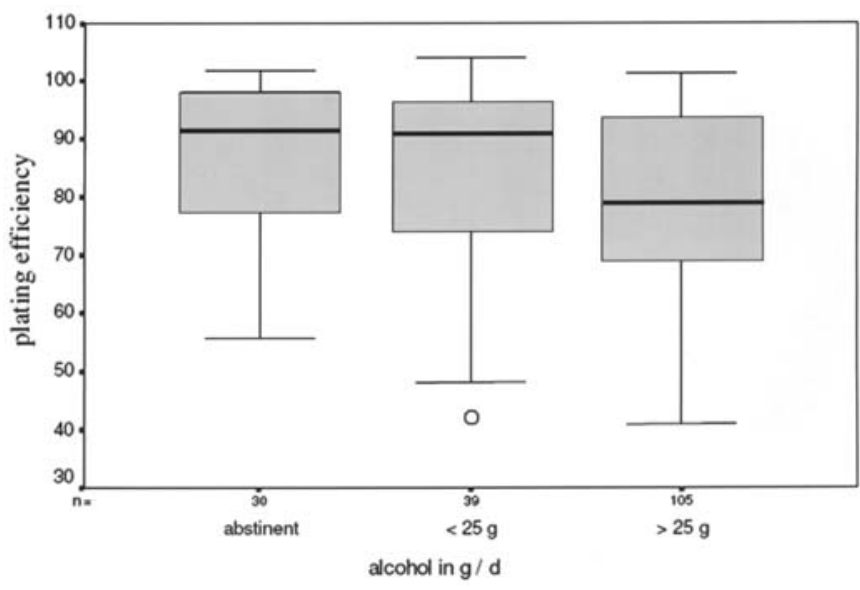

Figure 2. Boxplot diagram to visualise the reduction of plating efficiency in correlation to daily alcohol consumption. This slight tendency could not be proven with statistical methods (Mann-Whitney U test; Bonferroni testing; $\mathrm{p}>0.05)$. 


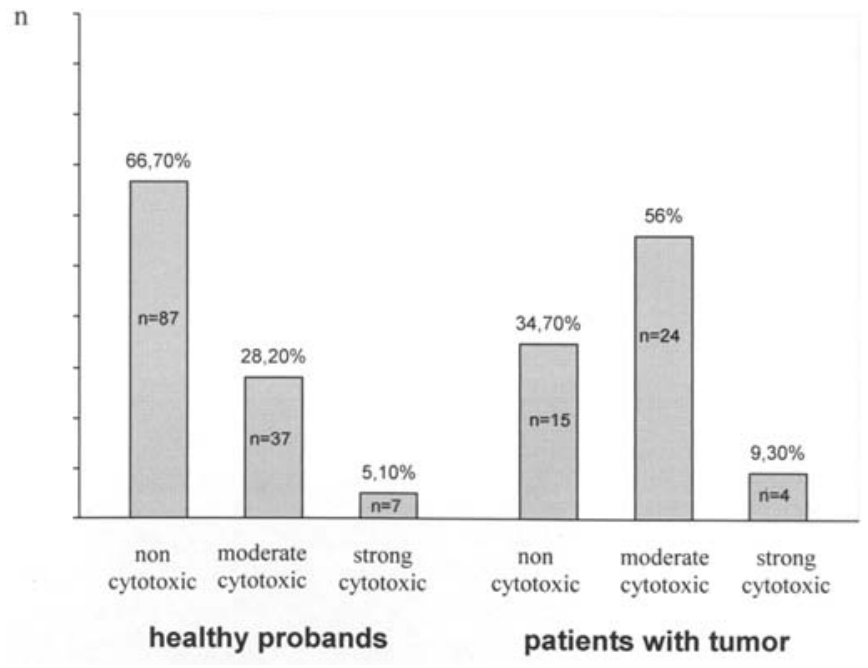

Figure 3. Cytotoxic effects in saliva samples of healthy probands and of patients with carcinomas of the upper aerodigestive tract.

Furthermore, we found increased cytotoxic effects in the saliva specimens of patients with carcinomas of the upper aerodigestive tract. The results are shown in Fig. 3. Due to the afore-mentioned cut-off points for defining plating efficiency of strong and moderate cytotoxicity, the difference between tumor patients and healthy probands was highly significant ( $\chi^{2}$ test; $p=0.001$ ). With regard to the aim of our study, we evaluated the plating efficiency biomarker as a tool for secondary prevention. Using logistic regression analysis, we estimated the relative cancer risk (odds ratio) of people with cytotoxic saliva to be 3.6 times higher than that of those without cytotoxic saliva (adjusted by tobacco consumption) $(\mathrm{p}<0.001)$.

\section{Discussion}

In the future, the incidence of SCC of the upper aerodigestive tract is likely to increase worldwide. This trend is already observable in many countries in Europe and North America $(2,3)$. Possible causes include changing lifestyle factors and exposure to and uptake of mutagenic substances. However, the import of different risk factors cannot be determined for individual human beings. Therefore, the identification and validation of biomarkers associated with the process of carcinogenesis may act as a potentially helpful tool for identifying individuals with increased cancer risk (14).

Cytotoxicity as a biomarker. In this study, we investigated the cytotoxic activity of saliva within a biomonitoring program to detect increased cancer risk. It is well known that biochemical or physiological variations in biological systems can lead to morphological reactions depending on the potency and duration of the influence (15). Hence, cytotoxic properties, in addition to the low-dose and long-term biological effects of exogenous noxious stimuli, should be measured through the identification of irreversible damage to different cellular components. Cumulative cytotoxic effects can cause malfunctions in the DNA repair system and changes in immunocompetence, and may directly incite genotoxic and carcinogenic reactions in tissues $(16,17)$.
Moreover, the death of tissue cells resulting from cytotoxicity leads to inflammation and hyperproliferation. The increase of the mitotic index favors the occurrence of persistent mutations in specific genomic locations - which are important for the regulation of the cell cycle (tumor suppressor genes and/or proto-oncogenes) - and may support carcinogenesis. This carcinogenic mechanism has been described in relation to non-genotoxic substances (e.g. saccharine) $(18,19)$. In summary, we can conclude that parameter 'cytotoxicity' characterises the biological tolerance of an organism to environmental exposure (20) and may also refer to increased carcinogenic activity.

It is generally agreed that the consumption of tobacco is the main risk factor for head and neck squamous cell carcinoma (HNSCC), and that this is due to its associated mutagenicity and cytotoxicity (15). A number of well-established methods exist to measure cytotoxic activity in biological samples using permanent cell cultures. Cytotoxicity, which is potentially based on genotoxic mechanisms, usually becomes effective after a time lag.

The measurement of the plating efficiency index in mammalian cell cultures takes these delayed effects into consideration. The chosen incubation time (7-10 days) of the cultured cells takes into account that they are able to repair acute damage via regenerative and adaptive mechanisms.

Relevance of saliva screening. The ease of sampling and handling the saliva of large populations has led to the question of whether saliva specimens can be usefully integrated into biological monitoring programs. Until now, saliva has been used very successfully for the chemical analysis of organic and inorganic substances (21). Etter et al measured increased cotinin concentrations in the saliva samples of smokers and passive smokers (22). The saliva of betel nut and tobaccochewing probands induced chromosomal alterations in mammalian cell cultures (23). Nevertheless, no previous study has evaluated the systemic cytotoxic potential of saliva specimens from healthy probands and patients with HNSCC.

The results of the examination of the 174 probands indicated a significant increase in the cytotoxic activity of the saliva specimens of smokers in comparison to non-smokers (Fig. 1). This finding must be interpreted as a systemic effect as the examined probands had smoked their last cigarette at least $12 \mathrm{~h}$ before the saliva sampling and had also performed their daily oral hygiene before the sampling period. Hence, the cytotoxic agents must have been secreted by the salivary glands. Interestingly, the results exhibited a significant difference in the cytotoxicity of lifelong non-smokers and former smokers, again providing evidence to support the presence of systemic effects. This fact suggests a persistent modified chemical composition of the saliva of former smokers. There are two conceivable reasons for this. First, lipophilic cytotoxic agents from inhaled tobacco smoke remain in the deep body compartment and are released over a long period of time in low-dose concentrations. The biological effects of these released substances, which are secreted within the saliva for years, were measured in our study. Second, this correlation may be due to a recall bias phenomenon. The chemical analysis of the saliva of former smokers is necessary if this question is to be answered. 
Despite the well-known function of alcoholic beverages as co-carcinogenic factors, we showed only a slight, statistically insignificant tendency of plating efficiency in relation to alcohol consumption (Fig. 2). A variety of reasons may be behind this unexpected result. Analogous to our former findings, it could be partly explained by a possible understatement of individual alcohol consumption by tumor patients in the interview (recall bias). Otherwise, the significant toxicological characteristics of tobacco components had a direct effect on cytotoxicity in comparison to the minor effect had by alcohol. Additionally, ethanol and acetaldehyde (the main degradation products), are quickly metabolised, depending on individual alcohol dehydrogenase activity, so only very recent alcohol consumption was detected using this method. Another unknown variable was the degree of bacterial colonisation in the oral cavity and gastrointestinal tract, which can directly influence the oxidation rate of ethanol (24).

Going back to the main focus of our study, we have evaluated the use of the plating efficiency index as a biomarker for future screening programs through its capacity to indicate increased individual cancer risk in the upper aerodigestive tract. Using logistic regression analysis, we found a 3.6-fold increased cancer risk in probands with cytotoxic saliva (Fig. 3).

Based on our results, the plating efficiency index can be used as a biomarker for exposure and effect, while also partly providing information on individual susceptibility (25). Hence, the advantages of in vitro biological testing of saliva lie in its detection of additional mutagenic effects caused by a multitude of specific substances with unknown toxicological thresholds, particularly in low-dose concentrations. Furthermore, the chemical composition of potentially mutagenic saliva is dependent on toxification and detoxification metabolic pathways that are influenced by different polymorphisms $(8,9)$. To what extent in vitro tests, like the plating efficiency index, illustrate carcinogenic phenomena in vivo remains controversial (26). However, it is beyond controversy that such in vitro techniques can help in understanding the stepwise malignant transformation of healthy tissue.

The combination of classical epidemiology with modern biological methods (so-called molecular epidemiology) will help in the detection of unknown cancer risk factors and may contribute to preventative measures against upper aerodigestive tract malignancies. This is important because, in the last decade, multimodal therapies have not significantly improved the survival rates of patients with head and neck cancer. In addition, current therapies are often mutilating and can permanently diminish the quality of life.

According to our results, the plating efficiency index is a suitable method for the detection of increased cancer risk. In combination with several effective biomarkers at cytogenetic end-points, it may help to establish biomonitoring programs for secondary cancer prevention (27). However, the plating efficiency index does not specify when or whether any malignant change will occur. Biomonitoring cannot replace medical examination and the histopathological diagnosis of suspect lesions. Nonetheless, it could become fundamental to the prevention of those cancers that are strongly influenced by exposure to environmental mutagens and endogenous risk factors (28-30).

\section{References}

1. Rudert H: Maligne Tumoren der Lippen, der Mundhöhle und des Oropharynx. In: Oto-Rhino-Laryngologie in Klinik und Praxis. Naumann HH, Helms J, Heberhold C und Kastenbauer E (eds). Thieme, Stuttgart, pp648-667, 2007.

2. La Vecchia C, Tavani A, Franceschi S, Levi F, Corrao G and Negri E: Epidemiology and prevention of oral cancer. Oral Oncol 33: 302-312, 1997.

3. Boyle P, Macfarlane GJ, Blot WJ, Ciesa F, Lefebre JL, Azul AM, Devries N and Scully C: European School of Oncology advisory report to the European Commission for the Europe Against Cancer Program - oral carcinogenesis in Europe. Eur J Cancer B Oral Oncol 31B: 75-85, 1995.

4. Egeli U, Ozkan L, Tunca B, Kahraman S, Cecener G, Ergul E and Engin K: The relationship between genetic susceptibility to head and neck cancer with the expression of common fragile sites. Head Neck 22: 591-598, 2000.

5. Schantz SP, Zhang ZF, Spitz MS, Sun M and Hsu TC: Genetic susceptibility to head and neck cancer; interaction between nutrition and mutagen sensitivity. Laryngoscope 107: 765-781, 1997.

6. Cloos J, Reid CB, Snow GB and Braakhuis BJ: Mutagen sensitivity: enhanced risk assessment of squamous cell carcinomas. Eur J Cancer B Oral Oncol 32: 367-372, 1996.

7. Copper MP, Jovanovic A, Nauta JJ, Braakhuis BJ, De Vries N, van der Waal I and Snow GB: Role of genetics in the etiology of squamous cell carcinoma of head and neck. Arch Otolaryngol Head Neck Surg 121: 157-160, 1995.

8. Matthias C, Jahnke V, Jones PW, Hoban PR, Alldersa JE, Worral SF, Fryer AA and Strange RC: Cyclin D1, glutathione S-transferase and cytochrome P450 genotype and outcome in patients with upper aerodigestive tract cancers: assessment of the importance of individual genes using multivariate analysis. Cancer Epidemiol Biomarkers Prev 8: 815-823, 1999.

9. Brockmöller J: Fremdstoffmetabolismus und Krebs: Molekularepidemiologische Forschung. In: Tumorerkrankungen. Handbuch der Molekularmedizin. Ganten D and Ruckpaul K (eds). Springer Verlag, Berlin, 1998.

10. Cheng TJ, Christiani DC, Xu X, Wain JC, Wiencke JK and Kelsey KT: Glutathione S-transferase genotype, diet, and smoking as determinants of sister chromatid exchange frequency in lymphocytes. Cancer Epidemiol Biomarkers Prev 4: 535-542, 1995

11. Hirvonen A, Nylund L, Kociba P, Husgafel-Pursiainen K, Vainino H: Modulation of urinary mutagenicity by genetically determined carcinogen metabolism in smokers. Carcinogesis 15: 813-815, 1994.

12. Carrano AV and Natarajan AT: Considerations for population monitoring using cytogenetic techniques. Mutat Res 204: 379-406, 1987.

13. Bradley MO, Bhuyan B, Francis MC, Langenbach R, Peterson A and Huberman V: Mutagenesis by chemical agents in V79 Chinese hamster cells: a review and analysis of the literature. A report of the Gene-Tox Program. Mutat Res 87: 81-142, 1981 .

14. IARC: Monographs on the evaluation the carcinogenic risk to humans. Solar and ultraviolet radiation. IARC, Lyon, pp551316, 1992.

15. Hinton DE and Larén DJ: Liver structural alterations accompanying chronic toxicity in fishes: potential biomarkers of exposure. In: Biomarkers of Environmental Contamination. McCarty JF and Shugart LR (eds). Lewis Publishers, Boca Raton, pp15-57, 1990.

16. Ashby J and Tennant RW: Chemical structure, Salmonella mutagenicity and extent of carcinogenicity as indicators of genotoxic carcinogenesis among 222 chemicals tested in rodents by the U.S.NCI/NTP. Mutat Res 204: 17-115, 1988.

17. Ashby $J$ and Tennant RW: Definitive relationships among chemicals, carcinogenicity and mutagenicity for 301 chemicals tested in rodents by the U.S.NTP. Mutat Res 257: 229-306, 1991.

18. Cohen SM and Ellwein L: Cell proliferation in carcinogenesis. Science 249: 1007-1011, 1990.

19. Cohen SM and Ellwein LB: Genetic errors, cell proliferation, and carcinogenesis. Cancer Res 51: 6493-6505, 1991.

20. Sverdrup LE, Kallqvist T, Kelley AE, Furst CS and Hagen SB: Comparative toxicity of acrylic acid to marine and freshwater microalgae and the significance for environmental effects assessment. Chemosphere 45: 653-658, 2001. 
21. Van Maanen JM, Welle IJ, Hageman G, Dallinga JW, Mertens PL and Kleinjans JC: Nitrate contamination of drinking water. Environ Health Perspect 104: 522-528, 1996.

22. Etter JF, Duc VT and Perneger TV: Saliva cotinine levels in smokers and non-smokers. Am J Epidemiol 151: 251-258, 2000.

23. Stich HF and Stich W: Chromosome-damaging activity of saliva of betel nut and tobacco chewers. Cancer Lett 15: 193$202,1982$.

24. Homann N, Jousimies-Somer H, Jokelainen K, Heine R and Salaspuro M: High acetaldehyde levels in saliva after ethanol consumption: methodological aspects and pathogenetic implications. Carcinogenesis 18: 1739-1743, 1997.

25. Indulski JA and Lutz W: Molecular epidemiology: cancer risk assessment using biomarkers for detecting early health effects in individuals exposed to occupational and environmental carcinogens. Rev Environ Health 12: 179-190, 1997.

26. Kleinsasser NH, Gamarra F, Bergner A, Wallner B, Harreus U, Juchhoff J, Kastenbauer E and Huber R: Genotoxicity of nitroso compouns and sodium dichromate in a model combining of human nasal epithelia and the comet assay. ORL J Otorhinolaryngol Relat Spec 63: 141-147, 2001.
27. Bloching M, Hofmann A, Lautenschläger C, Berghaus A and Grummt T: Exfoliative cytology of normal buccal mucosa to predict the relative risk of cancer in the upper aerodigestive tract using the micronucleus assay. Oral Oncol 36: 550-555, 2000.

28. Franceschi S, Barzan L and Talamini R: Screening for cancer of the head and neck: if not now, when? Oral Oncol 33: 313-316, 1997.

29. Bloching M, Stephan M, Agha-Mir-Salim P, Berghaus A, Lautenschläger C and Grummt T: Ames-test as biomarker. HNO 49: 440-446, 2001

30. Bloching M, Stephan D-U, Berghaus A, Lautenschläger C and Grummt T: In vitro determination of the cytotoxic activity of saliva samples of smokers. HNO 49: 630-635, 2001. 